\title{
La disfunción familiar y su repercusión en el adolescente. Barrio Rosa Mística - Paraguay, 2012
}

\author{
*Antonio Ulises Cusihuamán Puma ${ }^{1}$, Víctor Rodolfo Vert Gossen ${ }^{2}$, Julia Raquel Medina Rodríguez ${ }^{1}$, \\ Domingo Santiago Ávalos ${ }^{1}$, Marta Inés Ferreira Gaona ${ }^{3}$, Clarisse Virginia Díaz Reissner ${ }^{1,3}$ \\ ${ }^{1}$ Ministerio de Salud Pública y Bienestar Social. Paraguay \\ ${ }^{2}$ Instituto de Previsión Social. Paraguay \\ ${ }^{3}$ Universidad Católica Nuestra Señora de la Asunción. Paraguay
}

Cómo referenciar este artículo/

How to reference this article:

\begin{abstract}
Cusihuamán Puma AU, Vert Gossen VR, Medina Rodríguez JR, Ávalos DS, Ferreira Gaona MI, Díaz Reissner CV. La disfunción familiar y su repercusión en el adolescente. Barrio Rosa Mística - Paraguay, 2012. Mem. Inst. Investig. Cienc. Salud. 2018; 16(3): 22-29
\end{abstract}

\section{R E S U M E N}

El bienestar integral del adolescente está sujeto al entorno biopsicosocial de la familia. La disfunción familiar, como factor desequilibrante en la familia, se asocia con resultados negativos durante la adolescencia, tanto conductuales como de salud. Se realizó un estudio observacional descriptivo. El propósito fue describir la frecuencia de disfunción familiar y la repercusión en los adolescentes como grupo vulnerable en el seno familiar en el Barrio Rosa Mística, Mariano Roque Alonso en el 2012. Se utilizó el censo poblacional, identificándose los posibles riesgos biopsicosociales y familiares de la comunidad, la ficha clínica y el APGAR familiar como instrumentos válidos aplicados en un grupo de adolescentes residentes del Barrio Rosa Mística, área jurisdiccional de la Unidad de Salud Familiar "Virgen de Guadalupe" donde acudieron a las consultas médicas, previo consentimiento de los padres de familia y de los adolescentes. Fueron evaluados 148 adolescentes, la frecuencia de disfunción familiar severa fue de 2,0\% (APGAR familiar < 3), y la de disfunción familiar leve a moderada (APGAR familiar 4-7) 57,0\%, respectivamente. Existía disfunción familiar en este grupo de familias representativas (APGAR familiar leve a severo), pues se identificaron como factores de riesgo el grado de inestabilidad matrimonial de los padres de familia, la conformación del hogar, el embarazo en la adolescencia, la necesidad de trabajar del adolescente por motivos económicos lo que conlleva a la deserción escolar en algunos de los casos y, además, la alta probabilidad de que los adolescentes consuman alcohol y cigarrillo, repercutiendo en la salud biopsicosocial de los adolescentes.

Palabras clave: adolescente, factores de riesgo, APGAR familiar, atención primaria de salud.

\section{Family dysfunction and its impact in the adolescent Rosa Mística Neighborhood - Paraguay, 2012}

\section{A B S T R A C T}

The integral health of the adolescent is in a direct relationship to the biopsychosocial wellbeing of the family. Family dysfunction, as an unbalanced factor in the family, is associated with negative results during adolescence, both behavioral and health. An observational and descriptive study was carried out. The purpose was to describe the frequency of family dysfunction and the impact in the adolescents as a vulnerable group within the families of Rosa Mística neighborhood, Mariano Roque Alonso - 2012. A population census was used to identify the possible biopsychosocial and familiar risks of that community. The clinical history and the Family APGARs were used as valid instruments in a group of adolescent residents of Rosa Mística neighborhood which is into the jurisdiction of the "Virgen de Guadalupe Family Health Unit", where they attended for medical consultation, with previous consent of the adolescents and their parents. One hundred and 
forty eight adolescents were studied, the frequency of severe family dysfunction was $2.0 \%$ (Family APGAR $<3$ ) while $57.0 \%$ of mild and moderate family dysfunction (Family APGAR familiar 4-7). There was some kind of family dysfunction in these studied families (Family APGAR mild to severe). Risk factors identified were the degree of marital instability of the parents, family structure, adolescent pregnancy, need to work of the adolescents because of economic problems forcing them to give up school in some cases, high probability of adolescents to smoke or drink alcohol, all of these risk factors that affect the biopsychosocial health of the adolescents.

Keywords: adolescent, risk factors, family APGAR, primary health care.

\section{INTRODUCCIÓN}

La familia, núcleo de la sociedad, cubre las necesidades físicas de seguridad, de procreación, enseña destrezas socioculturales y fomenta propósitos para la vida a sus miembros, con el control de su ambiente y aceptación colectiva ${ }^{(1,2)}$.

El grado de salud familiar (organización, dinámica, funcionamiento) se determina mediante: a) el crecimiento familiar (morfogénesis); b) la estabilidad familiar (homeostasis); y c) la cohesión comportamental; representando ajustes y cambios requeridos en su organización y trayectoria de valores ${ }^{(3,4)}$.

El riesgo familiar es la probabilidad de consecuencias adversas individuales o familiares, de tipos biológico-demográficos, físico-ambientales, socioeconómicos, psicoafectivos, trayectoria familiar, prácticas de salud y servicios de salud ${ }^{(5)}$. En función del riesgo la estructura familiar se describe según: tipología (familia: nuclear ${ }^{(6)}$, nuclear modificada, nuclear reconstruida, extensa, extensa modificada, atípica); ciclo vital familiar; composición (número de personas, promedio de edad, sexo ${ }^{(3)}$.

EI APGAR familiar (elaborado por Smilkstein, 1978), ${ }^{(7)}$ contiene cinco enunciados que aluden a la familia funcional: Adaptación (A): Resolver problemas que amenazan el equilibrio familiar. Participación (P): Compartir la toma de decisiones y responsabilidades. Crecimiento (G - Growth): Madurez emocional, física y de autorrealización de los miembros con el soporte y guía mutua. Afecto (A): Relación de amor y atención mutua. Resolución (R): Compartir los recursos de espacio, tiempo y dinero ${ }^{(8)}$.

Al evaluar la disfunción familiar que afecta a los miembros de la familia ${ }^{(9)}$, el divorcio es un agravante para la pareja y los hijos. Al formar una etapa más del ciclo vital, se tiende a proteger a los hijos aun ante la desdicha ${ }^{(10)}$. El infortunio del divorcio para los hijos durante la infancia puede acarrear consecuencias en la salud mental en la adolescencia: déficit del rendimiento escolar y/o deserción, y violencia ${ }^{(11)}$. Deberían planificarse programas de contención y prevención para este tipo de población ${ }^{(12,13)}$.

El embarazo en la adolescencia, es otro problema álgido a la fecha con un aumento sostenido en su incidencia y requiere de estrategias vitales para prevenirlo ${ }^{(14)}$.

Luego está el fenómeno de transición demográfica (padres de familia que migran por plazas laborales y un mejor ingreso, aumenta el número de hogares monoparentales, y madres de familia que ingresan en la fuerza laboral) que torna endeble el núcleo protector del adolescente ${ }^{(15)}$. Y la transición epidemiológica (conductas de riesgo de los mismos adolescentes: abuso de sustancias, actividad sexual precoz, etc.) acarrea enfermedades de transmisión sexual, accidentes de tránsito, depresión y suicidio ${ }^{(5)}$.

Además, por la disgregación familiar u otros factores socioeconómicos, los hijos adolescentes se han visto impelidos a trabajar para salir adelante, inclusive abandonando los estudios, complicando aún más la realidad socioeconómica y cultural del individuo, de la familia y la sociedad ${ }^{(16)}$.

Las Unidades de Salud Familiar, en nuestro medio, procuran un mejor enfoque integral de los riesgos biopsicosociales del individuo, de la familia, y de la comunidad ${ }^{(17,18)}$.

Partiendo de estos conceptos, en la Unidad de Salud Familiar Virgen de Guadalupe, en el Barrio de Rosa Mística, Distrito de Mariano Roque Alonso, se quiso caracterizar el riesgo y el grado de salud de un grupo de adolescentes que conforman familias con cierto grado de disfunción familiar, buscando generar y plantear acciones preventivas de salud pública, en vista que no se han conocido estudios previos realizados en Paraguay que aborden la dimensión de dicha problemática desde Atención Primaria ${ }^{(19)}$.

Los objetivos del trabajo fueron determinar la frecuencia y caracterizar la disfunción familiar en los adolescentes como grupo vulnerable en el seno familiar, del Barrio de Rosa Mística, Mariano Roque Alonso - 2012. 


\section{MATERIALES Y MÉTODOS}

Se diseñó un estudio observacional descriptivo, transverso y retrospectivo, validado por el Comité de Ética en la Investigación de la Dirección General de Atención Primaria de la Salud. La unidad de muestreo y análisis fueron los adolescentes en 148 familias, de 10 a 18 años de ambos sexos, que habitan el Barrio Rosa Mística del Distrito de Mariano Roque Alonso, que acudieron al servicio en compañía de sus padres y que luego de ser atendidos en el consultorio médico accedieron a la entrevista, previo consentimiento informado de los padres de familia y de sus hijos adolescentes, respetándose los principios éticos de privacidad, intimidad, confidencialidad y anonimato. Cada uno de los encuestadores fue capacitado en la forma de instruir al encuestado ante las posibles eventuales solicitudes de aclaración por parte de los sujetos de estudio, utilizando el mismo patrón de cuestionarios. Se utilizó el censo poblacional que desarrolló la Unidad de Salud, identificándose los posibles riesgos biopsicosociales y familiares de la comunidad (información sobre vivienda, su ubicación y el número de personas que la conforman). En segundo lugar la historia clínica y el APGAR familiar según la técnica de Smilkstein $^{(7)}$ donde se considera cada uno de los cinco enunciados del instrumento en una escala de tres opciones: Siempre $=2$; Algunas veces $=1$; y Nunca $=0$, obteniéndose una puntuación del 0 al 10 , y así evaluar el posible grado de funcionamiento familiar (Buena función familiar: 8 - 10 puntos; Disfunción familiar leve a moderada: 4 - 7 puntos; y Disfunción familiar severa: 3 puntos o menos), lo cual se indagó durante las consultas médicas en el período de abril a septiembre de 2012.

Las variables incluidas fueron: demográficas, constitución y funcionamiento familiar, conductas de riesgo (violencia física o emocional), antecedentes personales de salud de los adolescentes, salud reproductiva, hábitos personales y familiares (alcoholismo y tabaquismo, consumo de sustancias), situación escolar de los adolescentes y situación laboral de los padres y de los adolescentes. Se interrogó por consumo de sustancias, conductas sexuales, síntomas emocionales y dinámica de comunicación familiar.

Los datos registrados fueron tabulados en una base diseñada en hoja electrónica Excel de Microsoft Corporation ${ }^{\mathrm{TM}}$ versión 2007. Las variables cualitativas se resumieron en frecuencias absolutas y porcentuales y se presentaron en tablas o gráficos, las cuantitativas en medidas de tendencia central y de dispersión. A pesar que el instrumento original (APGAR Familiar) puede ser aplicado a varios miembros de la familia, en este estudio se aplicó solamente a los adolescentes y se asoció la percepción de los mismos acerca de diversas dimensiones de la vida familiar, tales como funcionamiento, comunicación, cohesión, dinámica familiar: ausencia de tiempo para compartir en familia, ausencia de recursos o fuentes de apoyo ante la presencia de dificultades y una mala organización familiar ${ }^{(20)}$, y las variables dependientes (con excepción de variables del grupo "dinámicas de comunicación familiar") mediante la prueba de chi cuadrado de Pearson, expresándose la magnitud del riesgo en términos de odds ratio (OR).

\section{RESULTADOS}

Fueron evaluados 148 adolescentes, el $35,2 \%$ del sexo masculino, el $64,8 \%$ de sexo femenino; el promedio de edad fue de 14,2 años; el 3,4\% No Escolarizado y el $24,8 \%$ Escolaridad No acorde a la edad; el $64,8 \%$ con Tipología de Familia Nuclear (Tabla 1). 


\section{Tabla 1}

\begin{tabular}{|c|c|c|c|c|c|c|c|}
\hline \multirow[b]{2}{*}{ Variables } & & & \multicolumn{4}{|c|}{ Sexo } & \multirow[b]{2}{*}{ Valor $\mathrm{p}$} \\
\hline & \multicolumn{2}{|c|}{$\begin{array}{l}\text { General } \\
145(100 \\
\%)\end{array}$} & \multicolumn{2}{|c|}{$51(35,2 \%)$} & \multicolumn{2}{|c|}{$94(64,8 \%)$} & \\
\hline \multicolumn{8}{|l|}{ Edad } \\
\hline Media & \multicolumn{2}{|c|}{14,2} & \multicolumn{2}{|c|}{14,5} & \multicolumn{2}{|c|}{14,1} & 0,6 \\
\hline DE & \multicolumn{2}{|c|}{2,0} & \multicolumn{2}{|c|}{2,0} & \multicolumn{2}{|c|}{2,0} & \\
\hline Grupo & $\mathrm{N}^{\circ}$ & $\%$ & $\mathrm{~N}^{\circ}$ & $\%$ & $\mathrm{~N}^{\circ}$ & $\%$ & \\
\hline 10 a 12 & 36 & 24,8 & 10 & 19,6 & 26 & 27,7 & \\
\hline 13 a 15 & 70 & 48,3 & 24 & 47,1 & 46 & 48,9 & \\
\hline 16 a 18 & 39 & 26,9 & 17 & 33,3 & 22 & 23,4 & \\
\hline \multicolumn{8}{|l|}{ Escolarizado } \\
\hline SÍ & 140 & 96,6 & 48 & 94,1 & 92 & 97,9 & 0,2 \\
\hline NO & 5 & 3,4 & 3 & 5,9 & 2 & 2,1 & \\
\hline \multicolumn{8}{|l|}{ Escolaridad } \\
\hline Acorde edad & 114 & 78,6 & 35 & 68,6 & 79 & 84,0 & 0,03 \\
\hline No acorde & 31 & 21,4 & 16 & 31,4 & 15 & 16,0 & \\
\hline \multicolumn{8}{|l|}{ Tipología Familiar } \\
\hline Familia Nuclear & 94 & 64,8 & 32 & 62,7 & 62 & 66 & 0,54 \\
\hline Otra & 51 & 35,2 & 19 & 37,3 & 32 & 34 & \\
\hline
\end{tabular}

La frecuencia de disfunción familiar severa fue de 2,0\% (APGAR familiar <3). La disfunción familiar leve a moderada (APGAR familiar 4-7) se observó en el $57,0 \%$ de los casos (Figura 1).

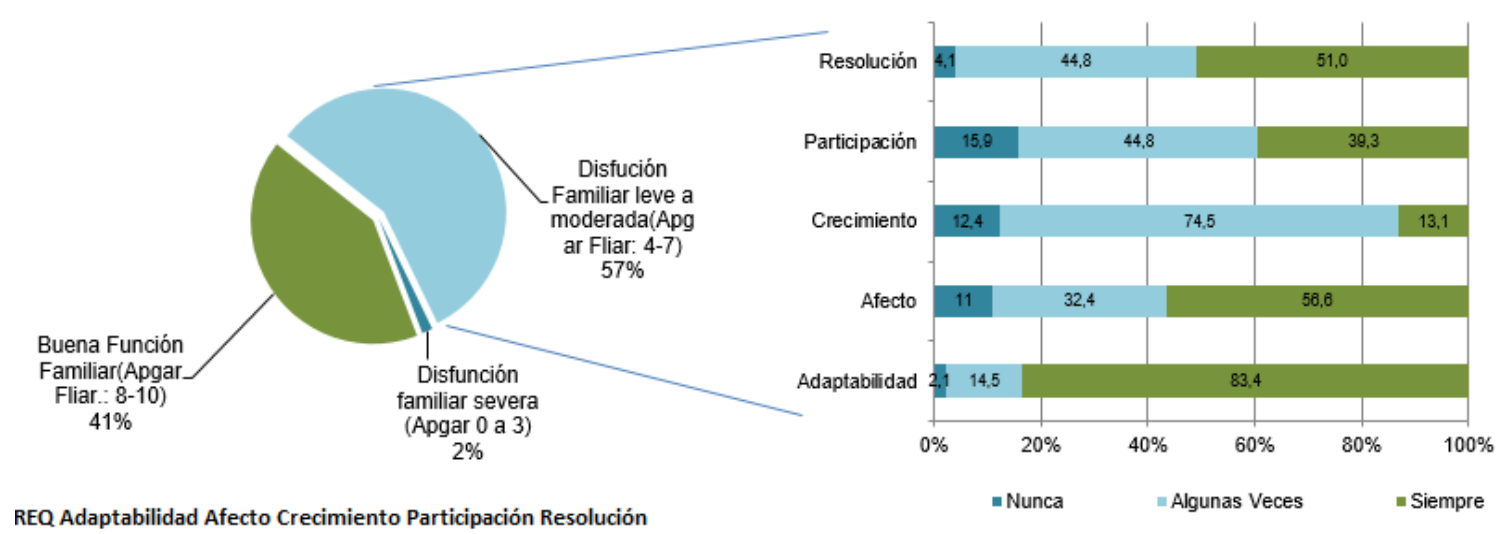

Figura 1

Uno de los posibles factores de riesgo familiar y social es el tipo de relación de los padres de familia (status matrimonial); en tal sentido se determinó que 103 adolescentes provenían de matrimonios estables $(69,6 \%), 9$ de uniones estables $(6,0 \%), 26$ convivían en con padres en proceso de separación $(17,6 \%), 2$ en situación de divorcio $(1,4 \%)$. (Figura 2). 


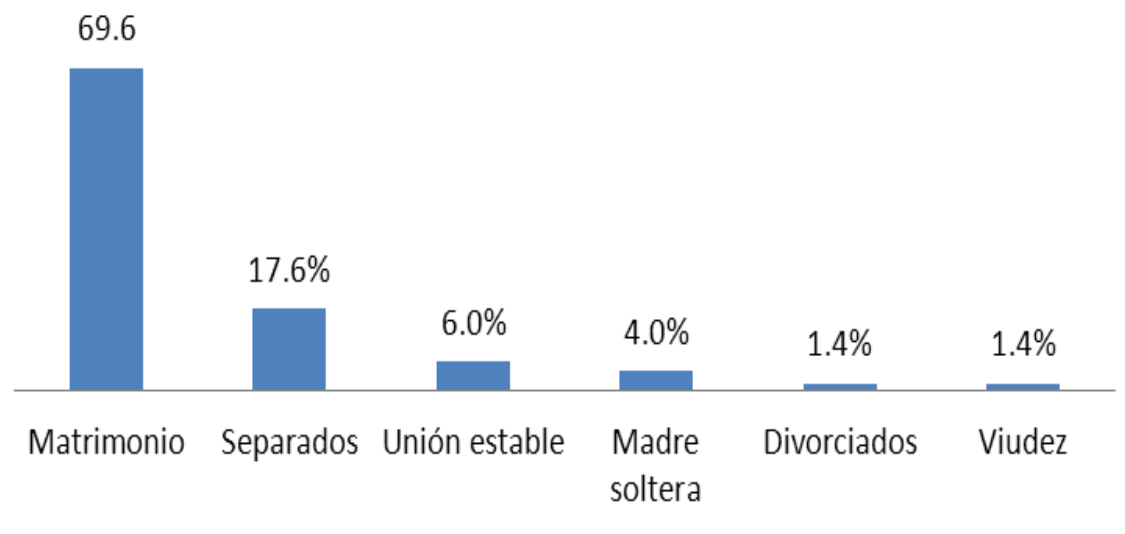

Figura 2: Status Matrimonial de los padres de los adolescentes encuestados. Barrio Rosa Mística-Paraguay $2012(n=148)$

La convivencia del adolescente con sus progenitores u otros familiares fue también evaluada. Se observó que $90(60,8 \%)$ vivían con ambos progenitores, $22(14,8 \%)$ sólo con uno de ellos, prevaleciendo la convivencia con sus madres. Además de convivir con la madre, $5(3,4 \%)$ también compartían con sus abuelas maternas, $10(6,8 \%)$ con el padrastro. Además, $13(8,9 \%)$ residían con otros familiares (abuelas, hermana, tíos, primos, y $6(4,0 \%)$ con su pareja sentimental (Figura 3$)$.

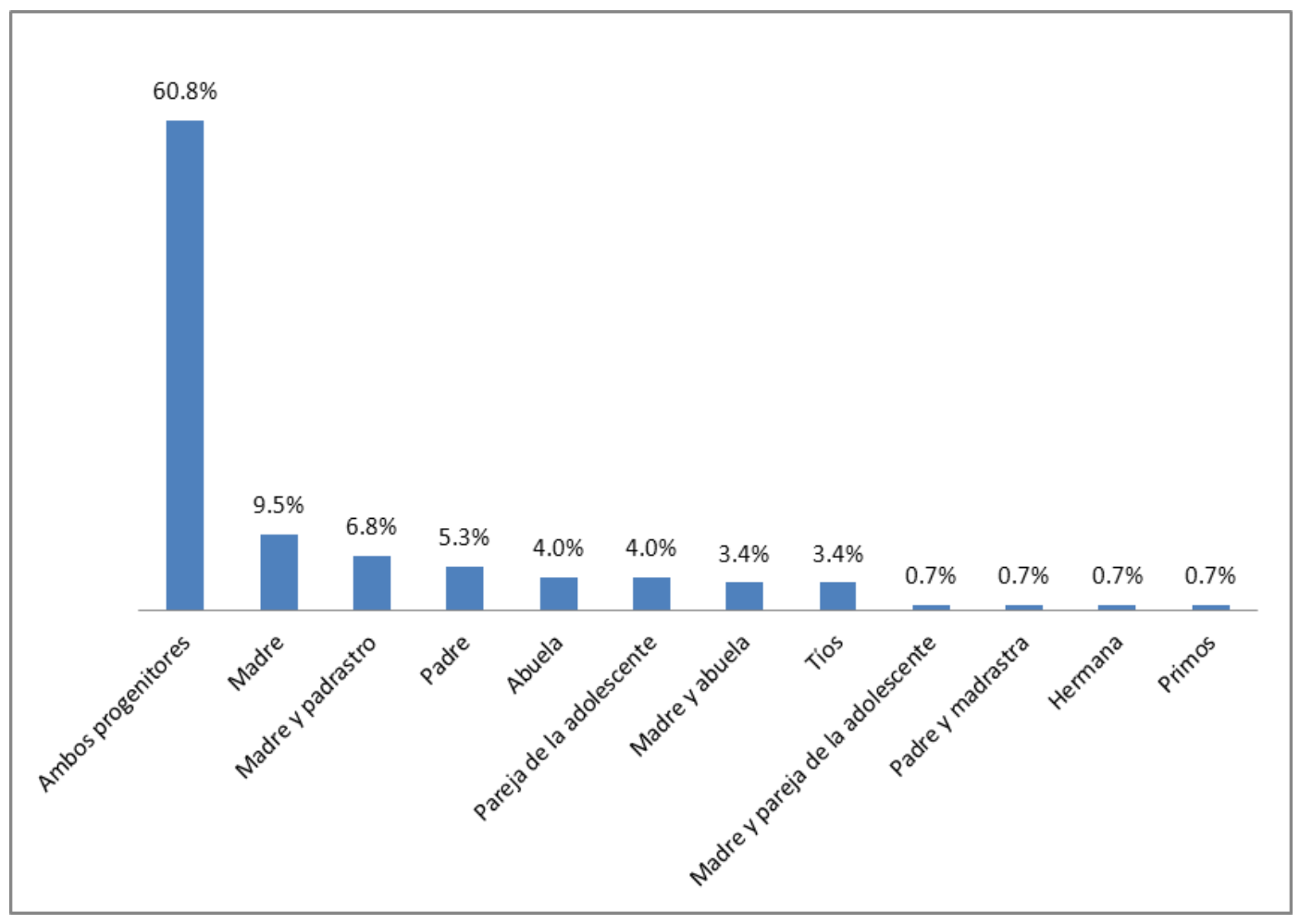

Figura 3. Convivencia de los adolescentes con sus progenitores u otros familiares. Barrio Rosa Mística - Paraguay, 2012. $(n=148)$

Nueve de las 102 adolescentes presentaban embarazos o ya tenían hijos, correspondiendo al 9,0\% del total de adolescentes mujeres. Lo cual no representaba una asociación directa con las familias disfuncionales ( $\mathrm{OR}=1,23 ; \mathrm{IC}=95 \%)$.

Del total de adolescentes encuestados el 2,0\% consumía sustancias tóxicas (alcohol y cigarrillo). En cuanto a la repercusión del consumo de sustancias por parte de los padres, no 
se encontró asociación con una mayor tendencia a consumir sustancias por parte de los hijos (OR=0,39; IC=95\%).

Finalmente, la deserción escolar representó el 3,4\% del total de adolescentes encuestados y los que trabajaban representaron el $5,4 \%$ del total de adolescentes encuestados (el 2,7\% trabajaban y estudiaban, y el 2,7\% dejaron de estudiar ante la necesidad de trabajar) (Figura 4). Se observó que la deserción escolar podría darse en relación a las familias disfuncionales $(\mathrm{OR}=2,12 ; \mathrm{IC}=95 \%)$.

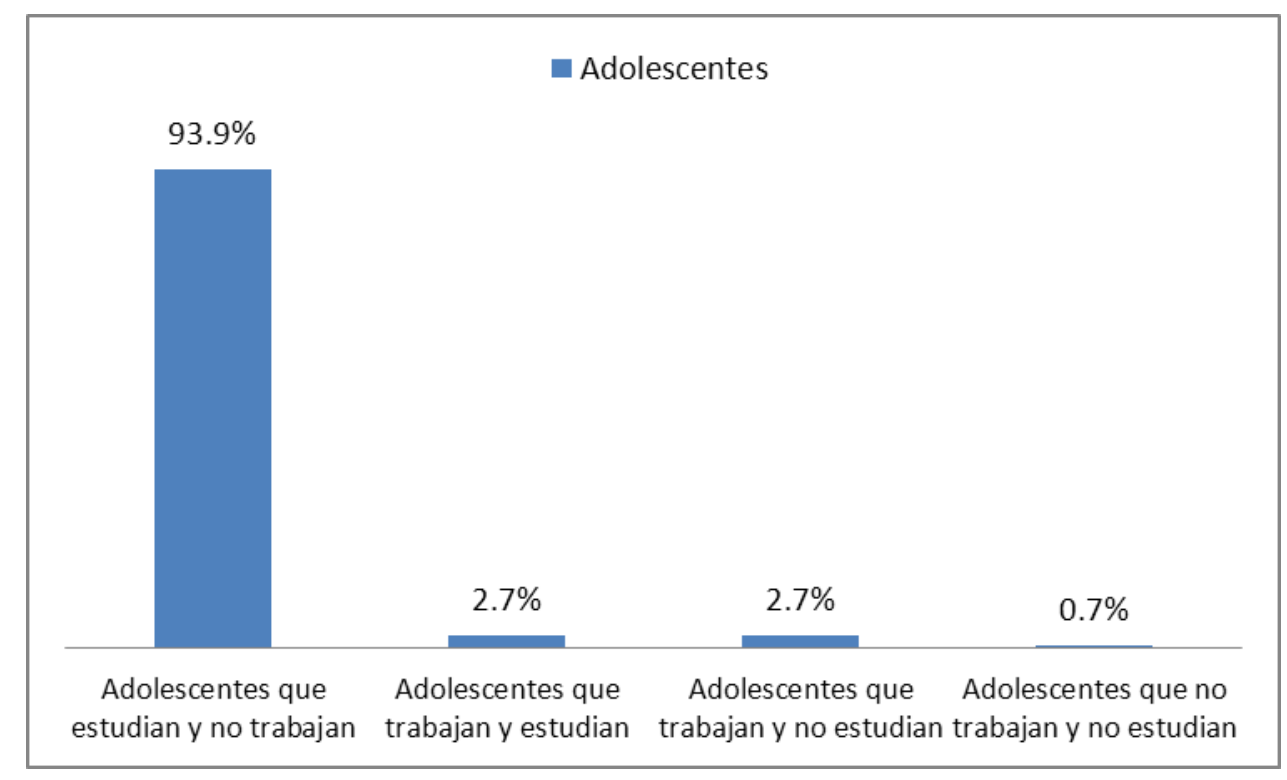

Figura 4: Adolescentes según situación laboral y/o deserción escolar. Barrio Rosa Mística Paraguay, 2012- $(n=148)$.

\section{DISCUSIÓN}

Según el estudio realizado a un grupo de 815 adolescentes provenientes de tres establecimientos educacionales de la Ciudad de Santiago de Chile, en junio del 2006, respondieron a un cuestionario validado por la OPS donde se exploró su percepción acerca funcionalidad y la dinámica familiar. Y se obtuvo que el $66,5 \%$ de los escolares percibía que sus familias eran disfuncionales.

Llama la atención que de todos los escolares chilenos integrantes de hogares percibidos por los estudiantes como disfuncionales resaltaba el hecho que presentaban un riesgo significativamente mayor del consumo de tabaco, marihuana, tranquilizantes, alcohol y cocaína. Por otro lado, menos del $50 \%$ de los adolescentes señalaba no tener una comunicación adecuada con sus progenitores. El 43,5\% de los adolescentes declaró haber tenido relaciones sexuales, siendo la prevalencia significativamente mayor en hombres que en mujeres.

El riesgo de presentar la totalidad de las condiciones descritas anteriormente fue significativamente mayor en adolescentes que percibían sus hogares como disfuncionales en comparación a hogares percibidos como funcionales ${ }^{(11)}$.

Otro estudio realizado en Cartagena, Colombia que se llevó a cabo el 2011 a un grupo de 1730 estudiantes entre hombres y mujeres de 13 y 17 años de edad que aceptaron participar, se identificó disfunción familiar mediante el APGAR familiar en un grupo de 896 estudiantes $(51,8 \%)$. Fueron predictores de disfunción familiar: síntomas depresivos con importancia clínica, baja religiosidad, familia no nuclear ${ }^{(21)}$, consumo de alguna sustancia en la vida, residente en estrato bajo y mal rendimiento académico ${ }^{(22)}$.

De esta manera, se puede visualizar que los factores de riesgo identificados y analizados en el presente trabajo afectan la dinámica y funcionalidad familiar y la salud del adolescente, aunque en menor escala en comparación al estudio obtenido en Santiago de Chile. Y el estudio de Cartagena se logró realizar a un grupo mayor de encuestados.

Existe disfunción familiar en este grupo de familias (APGAR familiar leve a severo), ya que los factores de riesgo identificados y analizados en el presente trabajo afectan la dinámica y funcionalidad familiar y la salud del adolescente. Resulta imprescindible que se 
cuente con el apoyo de un equipo multidisciplinario que trabaje en esta comunidad, brindando una atención diferenciada al adolescente ${ }^{(23)}$. Desde el punto de vista de prevención y promoción de la salud, incidir en la realización de charlas sobre salud sexual y reproductiva ${ }^{(24)}$, consumo de sustancias ${ }^{(25)}$. Y apoyo psicológico con un enfoque de rehabilitación. Involucrar a las instituciones educativas del medio, gobierno local y nacional (por citar, a través de la Secretaría de la Niñez y Adolescencia, de la Dirección de Salud Integral de la Niñez y la Adolescencia, y el Ministerio de Educación y Cultura), a la búsqueda de soluciones a corto y mediano plazo de los problemas identificados como factores de riesgo de la disfunción familiar de la comunidad.

Se recomienda efectuar otros estudios similares en las otras USF del territorio nacional con diferentes perfiles poblacionales a fin de poder establecer cuál es la situación general del país ${ }^{(26)}$, pudiendo abordarse, además, distintas problemáticas de salud que afecten al adolescente y que repercutan en la familia, a citar: abuso de sustancias ${ }^{(25)}$; relación sexual precoz que conlleven a embarazo en la adolescencia y/o infecciones de transmisión sexual $^{(27,28)}$, patología cervical ${ }^{(29)}$; depresión y riesgo de suicidio $^{(30)}$; bajo rendimiento escolar o deserción escolar ${ }^{(31)}$, entre otras.

Conflicto de intereses: Los responsables del presente trabajo no declaran ningún conflicto de interés con las instituciones ni los sujetos de estudio ya que los investigadores no han recibido remuneración alguna en tal sentido.

\section{REFERENCIAS BIBLIOGRÁFICAS}

1. Constitución de la República del Paraguay. Título II, Capítulo IV, Artículo 49-de la Protección de la familia. 1992. Disponible en: http://www.jme.gov.py

2. Friedemann ML. An instrument to evaluate effectiveness of family function. Western Journal of Nursing Research. 1991. 13:220-36.

3. Amaya de Peña P. Riesgo Familiar Total 770 y el ISF GEN 25. Referentes teóricosconceptuales y análisis de la información. Facultad de Enfermería, Universidad Nacional de Colombia. 2000.

4. Valdivia Sánchez C. Valores y familia ante el tercer milenio. Revista de Educación. España. 2001; 325:11-23.

5. Florenzano Urzúa $R$, Valdés Correa $M$. El Adolescente y sus conductas de riesgo. 3ra ed. Universidad Católica de Chile; 2005.

6. Organización de Naciones Unidas. Comisión Económica para América Latina y el Caribe (CEPAL), Notas de Población, No 99, (LC/G.2628-P), Santiago de Chile, 2014. Disponible en: http://www.un.org/es/development/progar eas/statistics.shtml

7. Good MJD, Smilkstein G, Good BJ. The family APGAR index: a study of construct validity. J Fam Pract 1979; 8: 55.

8. Alvarado Bestene J. Enfoque semiológico desde la medicina familiar, Introducción a la clínica. Centro Editorial Javeriano, CEJA. Bogotá, 2003.

9. Horwitz R. Familia y salud familiar. Un enfoque para la atención primaria. OPS, 1990.

10. Campell T, Bray J. The family's influence on health. Impact of divorce and remarriage on the family. En Rakel $R$, editor. Textbook of family practice. Saunders, 1995; 5:34-45.
11. Santander RS, Zubarew TG, Santelices LC, Argollo PM, Cerda JL, Bórquez MP. Salud y rendimiento académico. Factores $y$ conductas de riesgo en alumnos de pedagogía. Rev. méd. Chile 136(3) Santiago mar. 2008; 136: 317-24.

12. Breidablik $H$, Meland $E$. Experience of family break-up during childhood-health and health behavior in adolescence. Tidsskr Nor Laegeforen, 1999; 119:23315.

13. Steinman S, Petersen V. The impact of parental divorce for adolescents: A consideration of intervention beyond the crisis. Adolescent Med, 2001; 12:493-507.

14. Montes V, Álvarez C, Argote L, Osorio A. Gravidez en la adolescencia: Significado para la familia de hoy. Fam Saúde Desenv. 2006; 8(1):16-25.

15. Huitrón G, Denova E, Halley $E$, Santander S, Bórquez M, Zapata L. et al. Conductas de riesgo en una muestra de adolescentes chilenos y mexicanos: un estudio comparativo. Papeles de Población, México Med 17(70) oct-dic 2011; 17: 33-47.

16. Walsh F. Conceptualization of normal family functioning. Normal Family Process. Guillard Press, New York. 1982; 1:3-44.

17. Ministerio de Salud Pública y Bienestar Social de Paraguay (MSPBS). Manual de funciones de las unidades de salud de la familia. Asunción: MSPBS; 2006.

18. Correa AI, Herrera JA. La familia extensa y la función familiar. Colombia Med 1993; 24(1) 134-36.

19. Calvo $C$, Rubinstein Al. Influence of new evidence on medical practice. J Amb Board Fam Pract, 2002; 15:457-62.

20. Organización Panamericana de la Salud. Familia y Adolescencia. Indicadores de 
Salud. Manual de aplicación de instrumentos e instrumento abreviado. Washington D.C. 1999. Disponible en: http://www.paho.org/hq/index.php

21. Valdivia C. La Familia: concepto, cambios, nuevos modelos. La Revue du REDIF. Bilbao. 2008, 1:15-22.

22. Gómez E, Castillo I, Cogollo Z. Predictores de la disfunción familiar en adolescentes escolarizados. Revista de Psiquiatría. Colombia. 2013, 42:1.

23. Organización Panamericana de la Salud (OPS). Estrategia y plan de acción regional sobre los Adolescentes y Jóvenes 2010 2018. Disponible en:

http://new.paho.org/hq/dmdocuments/201 1/Estrategia-y-Plan-de-Accion-Regionalsobre-los-Adolescentes-y-Jovenes.pdf

24. Organización Panamericana de la Salud. IMAN servicios: normas de atención de salud sexual y reproductiva de adolescentes. Washington, DC: OPS; 2005.

25. Moral MV, Rodríguez FJ, Ovejero A. Correlatos psicosociales del consumo de sustancias psicoactivas en adolescentes españoles. Salud Publica Mex 2010; 52 (5): 406-15.

26. Franco-Giraldo A. El rol de los profesionales de la salud en la atención primaria en salud (APS). Rev. Fac. Nac. Salud Pública. Colombia 2015; 33(3): 41424.

27. Organización Panamericana de la Salud. Plan estratégico regional para el VIH/sida e
ITS, 2006-2015, de la Organización Panamericana de la Salud. Washington, DC: OPS; 2005. Disponible en: http://www.paho.org/spanish/ad/fch/ai/HI V_Regional_Plan_2006-

2015_SPANISH.pdf

28. Hurtado F, Donat F, Colomer J, Pla E, Sánchez A, Sarabia S, et al. Promoción, prevención, detección y actuación ante embarazos no deseados e infecciones de transmisión sexual en adolescencia desde Atención Primaria. C Med Psicosom. 2014;111. Disponible en: https://dialnet.unirioja.es/servlet/articulo? codigo $=4906940$

29. Organización Panamericana de la Salud. Estrategia y plan de acción regionales sobre la prevención y el control del cáncer cervicouterino en América Latina y $\mathrm{El}$ Caribe. Washington, DC: OPS; 2008. Disponible en: http://www.rho.org/files/PAHO_Regional_S trategy_2010_sp.pdf

30. Pavez P, Santander N, Carranza J, VeraVillarroel $P$. Familial risk factors for suicide among adolescents with depression. Rev. méd. Chile 2009; 137(2): 226-33.

31. Gutiérrez-Saldaña P, Camacho-Calderón N, Martínez-Martínez ML. Autoestima, funcionalidad familiar y rendimiento escolar en adolescentes. Aten Primaria. México. 2007;39(11):597-601. 